\title{
Large-Cavity Coronoids with Different Inner and Outer Edge Structures
}

Marco Di Giovannantonio, ${ }^{*}, \nabla$ Xuelin Yao, ${ }^{\nabla}$ Kristjan Eimre, ${ }^{\nabla}$ José I. Urgel, Pascal Ruffieux, Carlo A. Pignedoli,* Klaus Müllen,* Roman Fasel, and Akimitsu Narita*

Cite This: J. Am. Chem. Soc. 2020, 142, 12046-12050

Read Online

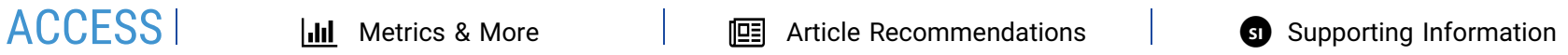

ABSTRACT: Coronoids, polycyclic aromatic hydrocarbons with geometrically defined cavities, are promising model structures of porous graphene. Here, we report the on-surface synthesis of C168 and C140 coronoids, referred to as [6]- and [5]coronoid, respectively, using 5,9-dibromo-14-phenylbenzo $[\mathrm{m}]$ tetraphene as the precursor. These coronoids entail large cavities $(>1 \mathrm{~nm})$ with inner zigzag edges, distinct from their outer armchair edges. While [6]coronoid is planar, [5]coronoid is not. Low-temperature scanning tunneling microscopy/spectroscopy and noncontact atomic force microscopy unveil structural and electronic properties in accordance with those obtained from density functional theory calculations. Detailed analysis of ring current effects identifies the rings with the highest aromaticity of these coronoids, whose pattern matches their Clar structure. The pores of the obtained coronoids offer intriguing possibilities of further functionalization toward advanced host-guest applications.

C oronoids are macrocyclic conjugated hydrocarbons formed by circularly fused benzenoid rings, featuring a cavity. ${ }^{1-3}$ Kekulene is a representative coronoid with a single layer of benzene rings around the cavity, belonging to the family of cycloarenes. The first synthesis of kekulene in $1978^{4}$ provided fundamental information about the nature of macrocyclic conjugation and aromaticity, pointing out that kekulene is better represented by Clar's model rather than the so-called Kekule structure. Other examples of cycloarenes are cyclo[d,e,d,e,e,d,e,d,e,e]decakisbenzene, ${ }^{5}$ predicted to have intriguing electronic and magnetic properties, ${ }^{6-9}$ septulene, ${ }^{10}$ and octulene. ${ }^{11}$ In 2016, we reported a C216 coronoid, ${ }^{1}$ a macrocyclic molecule with $216 \mathrm{sp}^{2}$ carbon atoms distributed in more than one layer of circularly fused benzene rings (Figure 1). The C216 coronoid can also be regarded as a nanographene with a cavity arising upon removal of the central benzene ring from a C222 nanographene (Figure 1). Detailed characterization of C216 was compromised by its vanishing solubility, which also limited further synthetic studies of large coronoids. Very recently, Tan and co-workers reported a synthesis of mesityl-substituted $\mathbf{C 1 0 8}$ coronoid, which formed molecular bilayers. $^{12}$

On the other hand, on-surface chemistry offers an alternative method to synthesize nanographenes and to explore their structure and electronic properties via state-of-the-art scanning probe microscopy and spectroscopy. ${ }^{13-15}$ Recently, kekulene was studied using noncontact atomic force microscopy (ncAFM), which corroborated its structure in accordance with Clar's model. ${ }^{16}$ Additionally, the on-surface synthesis of a C108 coronoid (Figure 1) was achieved, ${ }^{17}$ which represents a smaller homologue of the $\mathbf{C 2 1 6}$ coronoid. However, to date, no other coronoids have been synthesized on surfaces.

Coronoids can also serve as models for graphene with nanoscale cavities, namely nanoporous graphene, which, depending on the size and structure of the pores, has intriguing

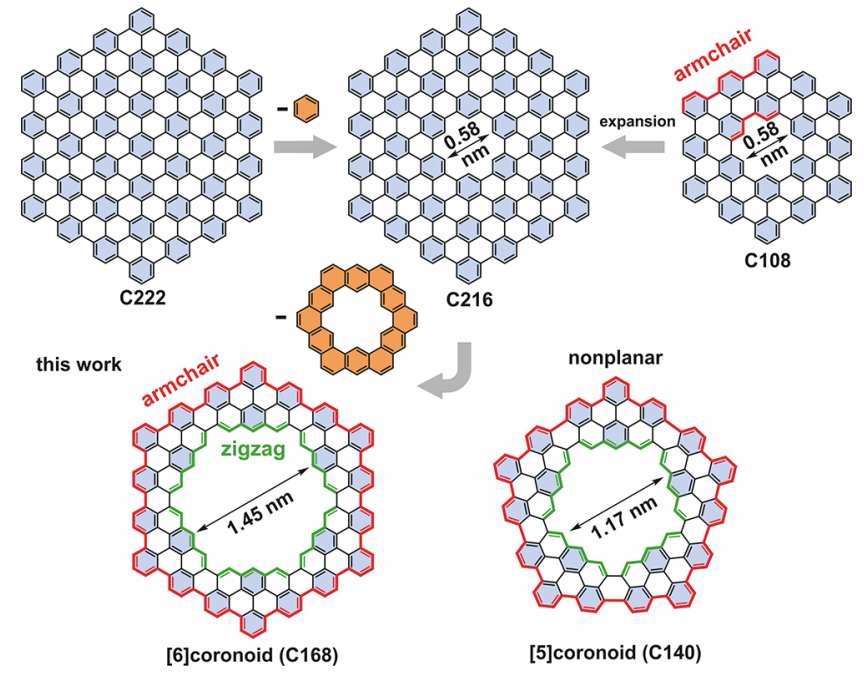

Figure 1. From nanographenes to coronoids. Clar sextets are filled in light blue. See Figure S3 for pore size determination.

electronic and magnetic properties. ${ }^{18-20}$ Nanoporous graphene with zigzag-edge cavities stands out ${ }^{21}$ since it is related to nanographenes and graphene nanoribbons with zigzag edges. $^{22,23}$ However, all reported coronoids have inner and outer armchair edges, and coronoids hosting a cavity with zigzag edges have never been synthesized.

Received: May 13, 2020

Published: June 26, 2020 

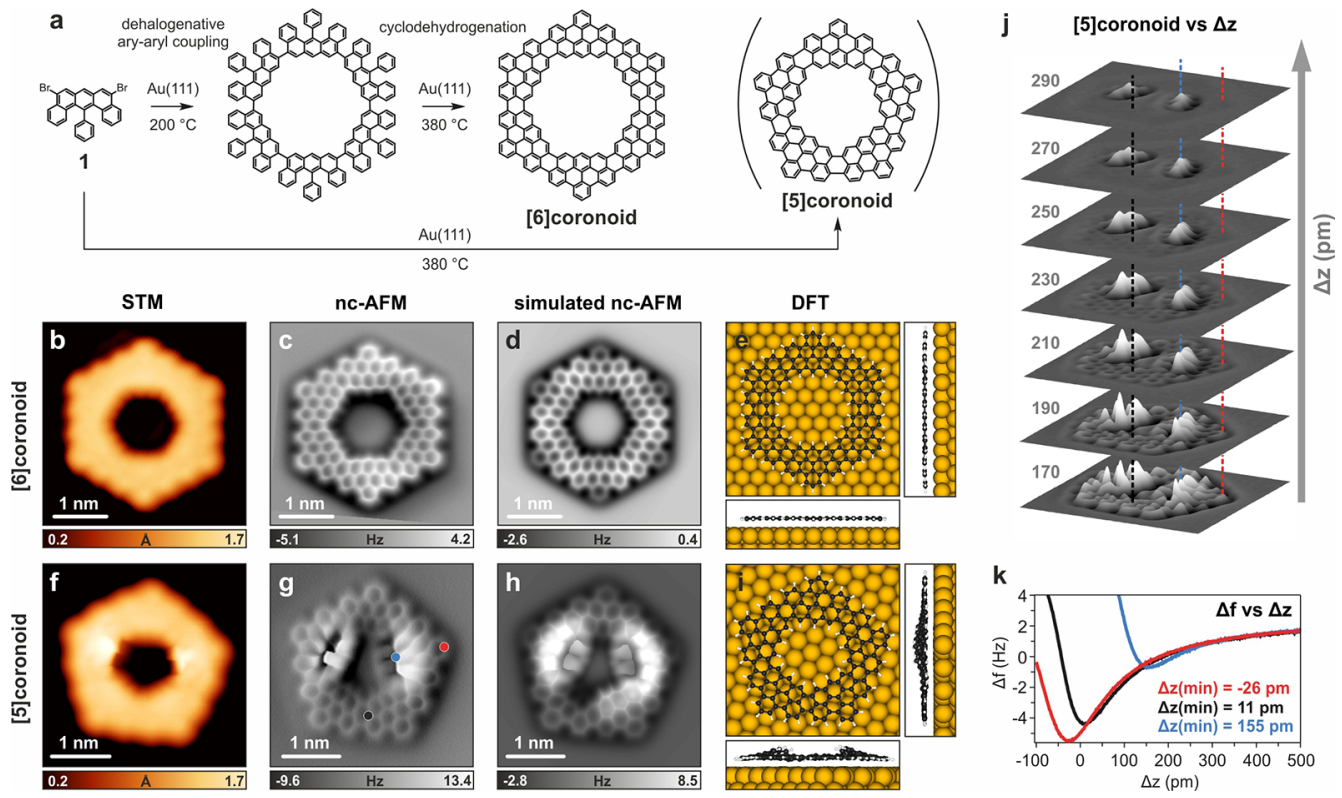

Figure 2. On-surface synthesis and characterization of [6]- and [5]coronoids. (a) Synthetic scheme. (b,f) High-resolution STM images. (c,g) ncAFM images acquired with a CO-functionalized tip. (d,h) Simulated nc-AFM images of the DFT-optimized structures shown in panels (e,i). (j) 3D view of tip height $(\mathrm{z})$ dependent nc-AFM images of [5] coronoid. (k) Frequency shift $(\Delta f)$ vs $\Delta z$ curves at different molecular sites (the colors of the curves correspond to those of the dots and the dashed lines in panels $\mathrm{g}$ and $\mathrm{j}$, respectively). Scanning parameters: $(\mathrm{b}) V_{\mathrm{b}}=-20 \mathrm{mV}, I_{\mathrm{t}}=100$ pA; (c) $\Delta \mathrm{z}=+150 \AA$ with respect to STM set point: $V_{\mathrm{b}}=-5 \mathrm{mV}, I_{\mathrm{t}}=100 \mathrm{pA}$; (f) $V_{\mathrm{b}}=-20 \mathrm{mV}, I_{\mathrm{t}}=100 \mathrm{pA}$; $(\mathrm{g}) \Delta \mathrm{z}=+170 \AA$ with respect to STM set point: $V_{\mathrm{b}}=-5 \mathrm{mV}, I_{\mathrm{t}}=100 \mathrm{pA} ;(\mathrm{j}) \Delta z$ with respect to $S T M$ set point $\left(V_{\mathrm{b}}=-5 \mathrm{mV}, I_{\mathrm{t}}=100 \mathrm{pA}\right)$ is indicated for each layer.

In this work, we targeted an exemplary coronoid (C168) with outer armchair edges and a large pore $(1.45 \mathrm{~nm}, \mathrm{C}-\mathrm{C}$ distance) featuring inner zigzag edges. Conceptually, this molecule can be obtained via removal of a kekulene from the aforementioned C216 coronoid (Figure 1). Using 5,9dibromo-14-phenylbenzo $[m]$ tetraphene $(1)$ as a precursor, we achieved a thermally activated on-surface synthesis of the C168 coronoid through dehalogenative aryl-aryl coupling and subsequent cyclodehydrogenation on $\mathrm{Au}(111)$ (Figure 2a). C168 is obtained by the coupling of six molecules 1 and is hereafter called [6]coronoid. The on-surface synthesis also led to the formation of a coronoid consisting of five molecules $\mathbf{1}$, namely C140 ([5]coronoid), which adopts a strain-driven nonplanar conformation. The on-surface structural and electronic characterization of these coronoids was performed by scanning tunneling microscopy/spectroscopy (STM/STS) and nc-AFM, which were supplemented by density functional theory (DFT) calculations supporting the experimental results and shedding light on the aromaticity of [5]- and [6]coronoids.

For the synthesis of [6] coronoid we have designed the Ushaped precursor $\mathbf{1}$, having a benzo $[\mathrm{m}]$ tetraphene core with a preinstalled zigzag edge as well as two bromo groups carefully positioned to allow for the macrocyclization on surface (see the SI for the synthesis of characterizations of $\mathbf{1}$ ). When $\mathbf{1}$ was deposited onto a $\mathrm{Au}(111)$ surface held at $200{ }^{\circ} \mathrm{C}$, the homolytic cleavage of $\mathrm{C}-\mathrm{Br}$ bonds readily took place, initiating aryl-aryl coupling. ${ }^{24}$ A temperature increase to $380{ }^{\circ} \mathrm{C}$ promoted the cyclodehydrogenation of the obtained nanostructures. $^{25-27}$ The final products of this sequence consisted of various chainlike structures, as well as some macrocyclic molecules (Figure S1) including highly symmetrical ones (Figure 2b,f). The yields of [5]- and [6] coronoids could be increased by applying high-dilution conditions, ${ }^{28}$ with the highest yields of $30 \%$ and $6 \%$, respectively, when the deposition rate of 1 onto $\mathrm{Au}(111)$ was set at 0.23 monolayer/hour (see Figure S2 for further details). The higher yield of [5] coronoid is most likely due to the ability of pentameric intermediates to undergo intramolecular head-totail coupling before the incorporation of the sixth building block, prevented by the high-dilution conditions.

To assess the exact chemical structure of the observed macrocycles, we acquired constant-height frequency-shift ncAFM images using a CO-functionalized tip, ${ }^{29}$ which clearly revealed the formation of [6]- and [5] coronoid (Figure 2c,g). [6]Coronoid is planar on the $\mathrm{Au}(111)$ surface and displays the expected armchair/zigzag configurations of its outer/inner edges, as further confirmed by the excellent agreement of the experimental results with simulated nc-AFM images (Figure $2 \mathrm{~d}$ ) based on the DFT-optimized structural model (Figure 2e). The size of the inner cavity is measured to be $1.40 \pm 0.05 \mathrm{~nm}$ (C-C distance of two opposing zigzag edges), in accordance with the DFT-calculated value of $1.45 \mathrm{~nm}$ (see the SI for details). The [5]coronoid also reveals an armchair/zigzag configuration of outer/inner edges (Figure 2f,g), while the size of the inner cavity is reduced to $1.10 \pm 0.05 \mathrm{~nm}$ (diameter of the inscribed circle through the central carbon atoms of the zigzag edges measured from the nc-AFM data). Again, this value agrees with the pore size of $1.09 \mathrm{~nm}$ estimated from the DFT-optimized geometry of the [5] coronoid on $\mathrm{Au}(111)$ (Figure 2i). For the [5] coronoid, two out of five inner edges appear as brighter/higher features in both the STM and ncAFM images (Figure 2f,g). Such a nonplanar structure is in line with a partial flattening of the more distorted gas-phase geometry of [5] coronoid (Figure S3b) upon adsorption. For quantification, we acquired $z$-dependent nc-AFM images (Figure $2 \mathrm{j}$ ) and recorded the frequency shift $(\Delta f)$ of the CO-functionalized tip while it approached the molecule to a specific location (Figure 2k). This allowed us to extract a height difference between the highest (blue) and lowest (red) 
part of the molecule of $180 \pm 10 \mathrm{pm}$, in good agreement with a DFT-calculated value of $170 \mathrm{pm}$ (determined from the optimized geometry in Figure 2i). This height difference is substantially smaller than the value calculated for the [5]coronoid in the gas phase $(360 \mathrm{pm}$, Figure S3b), which reflects the effect of van der Waals interactions with the $\mathrm{Au}(111)$ surface upon flattening the molecule.

To characterize the electronic properties of the [5]- and [6] coronoids, we performed constant-height differential conductance $\mathrm{d} I / \mathrm{d} V$ measurements and constant-current $\mathrm{d} I$ / $\mathrm{d} V$ mappings at the detected positive (PIR) and negative ion resonances (NIR) (Figure $3 \mathrm{a}-\mathrm{j}$ ). Gas-phase orbital calcu-
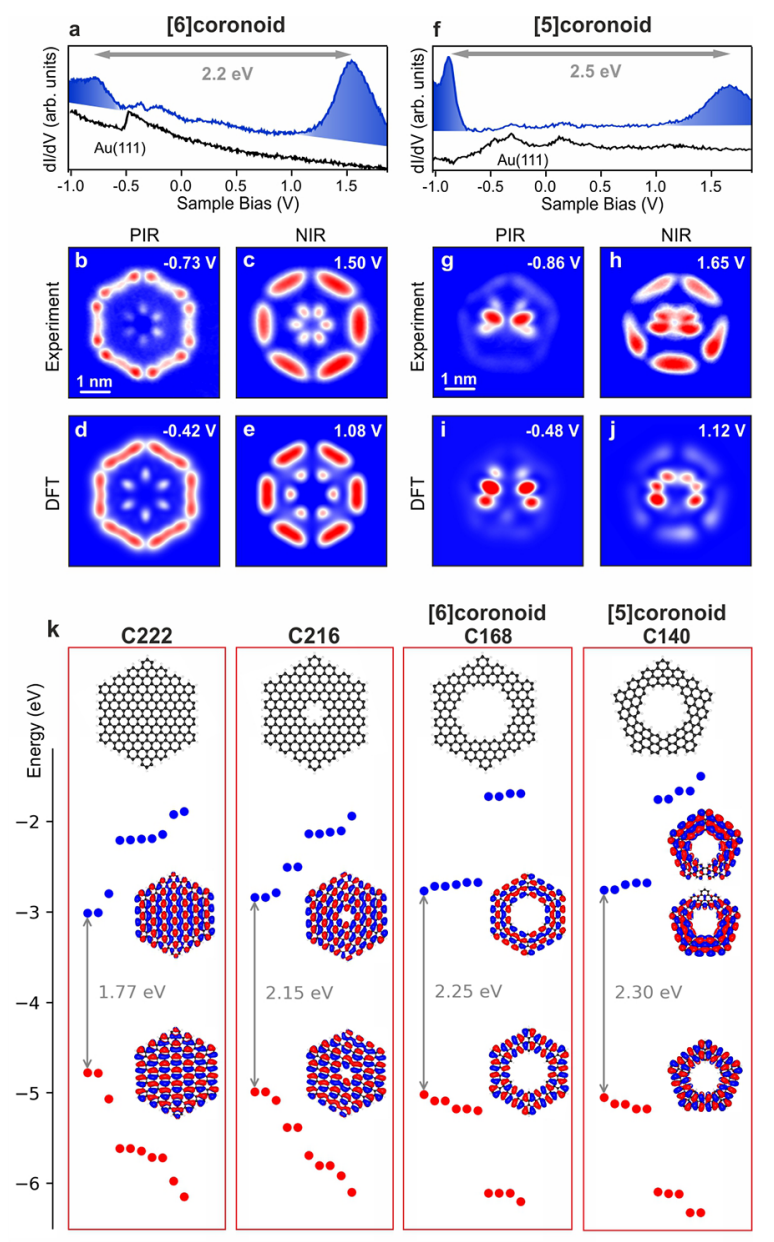

Figure 3. Differential conductance measurements and DFT simulations of [6]- and [5] coronoids. (a,f) dI/dV spectra. (b,c,g,h) dI/dV maps. (d,e,i,j) DFT-calculated local density of states at the frontier PDOS peaks (see Figure S4) of the two systems on $\mathrm{Au}(111$ ) at the PBE level of theory. (k) Gas phase electronic characterization of the four indicated systems at the B3LYP/6-311+G** level of theory. The red and blue dots represent the occupied and unoccupied states, respectively The HOMO and LUMO wave functions are displayed. For the [5]coronoid, the LUMO is doubly quasidegenerate and both orbitals are shown.

lations (Figure 3k) show for [6]- and [5]coronoid, respectively, 6 and 5 close-lying frontier orbitals both for occupied and unoccupied levels. This is reflected in DFT calculations of the molecules on $\mathrm{Au}(111)$, where both the HOMO and LUMO are hybridized with the respective closelying molecular orbitals into singular frontier ion resonances (see Figure S4 for details) that can be assigned to the experimentally measured PIR and NIR signatures. Hence, the experimental HOMO-LUMO gaps of the [6]- and [5]coronoids on $\mathrm{Au}(111)$ are 2.2 and $2.5 \mathrm{eV}$, respectively. DFT calculations predicted the gas-phase HOMO-LUMO gaps of the [5]- and [6]coronoid, C216 and C222, to be 2.30, 2.25, 2.15 , and $1.77 \mathrm{eV}$, respectively (Figure $3 \mathrm{k}$ ), highlighting the trend of increasing gap with increasing pore size and decreasing number of carbon atoms.

The [6]coronoid could potentially constitute a building block for extended nanoporous graphene with larger cavities than those reported so far ${ }^{20,30-32}$ and thus be of interest in the framework of graphene antidot lattices. ${ }^{33}$ We calculated the electronic properties of such hypothetical periodic layer via tight binding (TB) simulations (see Figure S5 for details), which reveal a band gap of $0.63 \mathrm{eV}$ (between frontier bands localized at each cavity rim), considerably reduced compared to the [6] coronoid HOMO-LUMO gap (TB) of $1.51 \mathrm{eV}$.

Carbon macrocycles are known to have peculiar aromaticity properties, leading to intriguing concepts, such as superaromaticity, ${ }^{7,34}$ and represent an ideal playground for studying fundamental aspects of the $\pi$-electron clouds. To display the aromaticity pattern of the [6]- and [5]coronoid, we performed nucleus-independent chemical shift (NICS) calculations at $1 \AA$ above the center of each carbon ring $\left(\operatorname{NICS}_{z z}(1)\right.$, Figure $\left.4 a, d\right)$. The results qualitatively match between similar locations of [6]- and [5]coronoids, indicating that the nonplanarity of the
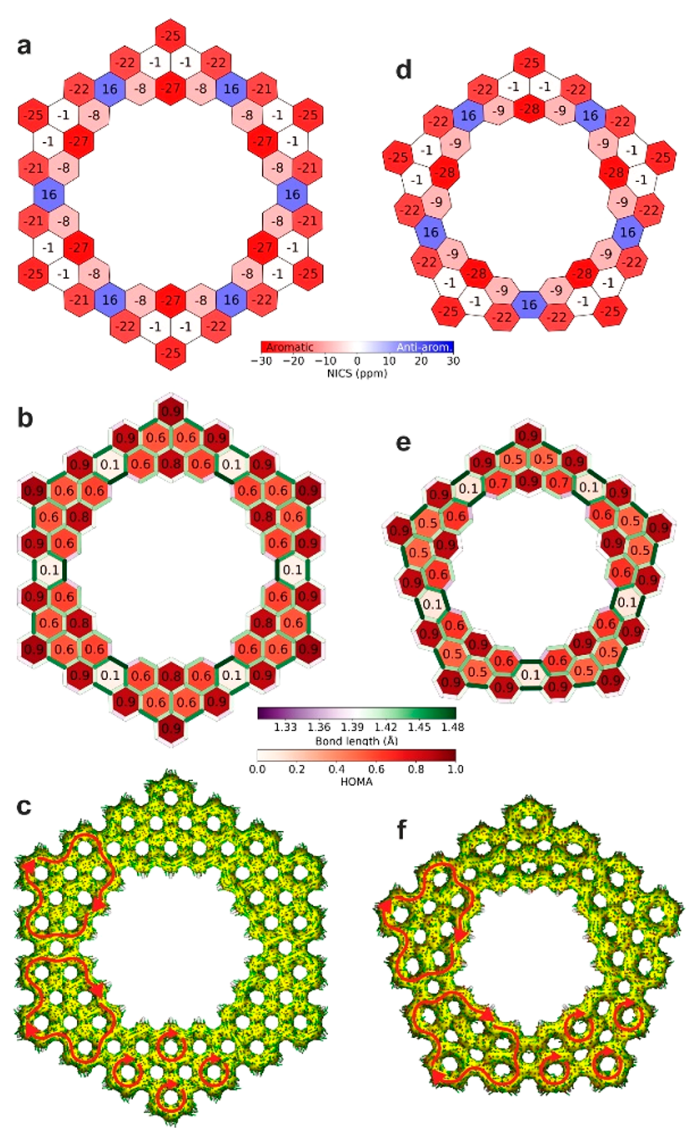

Figure 4. Aromaticity of [6]- and [5]coronoid. (a,d) $\operatorname{NICS}_{z z}(1)$ patterns. (b,e) HOMA analysis. (c,f) ACID analysis (isovalue $0.05 \mathrm{au}$ ) and currents induced in the delocalized electrons. Red arrows show diatropic currents. All calculations performed at the B3LYP/6$311+\mathrm{G}^{* *} / / \mathrm{B} 3 \mathrm{LYP} / 6-311 \mathrm{G}^{* *}$ level of theory. 
latter does not significantly affect its aromaticity. Moreover, the $\mathrm{NICS}_{z z}(1)$ patterns resemble the Clar structure of these coronoids (compare Figures 1 and 4), with each Clar sextet showing strong aromatic character (from $-22 \mathrm{ppm}$ to -27 $\mathrm{ppm})$, similar to benzene $(-29 \mathrm{ppm})$. The hexagons connecting the aromatic phenanthro[2,3,4,5-pqrab] perylene substructures (at the "bridges" between the fused precursor units) exhibit positive NICS value (16 ppm, Figure 4a,d) reminiscent of perylene. ${ }^{35}$ In this regard, the [6]- and $[5]$ coronoids can be well represented by alternatingly fused perylene and pyrene constituents (Figure S6). To shed further light on the observed aromaticity pattern, we performed a harmonic oscillator model of aromaticity (HOMA) analysis (Figure 4b,e), which qualitatively matches the $\operatorname{NICS}_{z z}(1)$ patterns of [6]- and [5]coronoid. Further, we studied the induced currents due to an applied magnetic field by analyzing the anisotropy of the induced current density (ACID) (Figure $4 c, f)$. The valence electrons are delocalized throughout the [6]- and [5]coronoids. Each phenanthro[2,3,4,5-pqrab]perylene substructure displays a macroscopic diatropic current on its outer edge (red arrows in Figure 4c,f). Within these substructures, the most aromatic sextets exhibit local diatropic currents.

In conclusion, we have achieved the successful on-surface synthesis of large, planar [6]- and nonplanar [5]coronoids featuring outer armchair and inner zigzag edges. Our study provides a complete characterization of their fundamental structural and electronic properties, revealing a HOMOLUMO gap of 2.2 and $2.5 \mathrm{eV}$ on $\mathrm{Au}(111)$, respectively. Both coronoids present similar aromaticity pattern and homogeneously distributed $\pi$-electron clouds, and the location of the most aromatic sextets matches their Clar structure. The [6]and [5] coronoid have unprecedentedly large pores of $1.40 \pm$ 0.05 and $1.10 \pm 0.05 \mathrm{~nm}$, respectively. Our synthetic strategy opens new avenues toward coronoids with diversified outer and inner edge structures and offers a rich playground to investigate fundamental electronic properties of coronoids. Further, lateral fusion of these coronoids potentially leads to nanoporous graphene.

\section{ASSOCIATED CONTENT}

\section{SI Supporting Information}

The Supporting Information is available free of charge at https://pubs.acs.org/doi/10.1021/jacs.0c05268.

Methods, precursor synthesis and characterizations, additional computational details, and additional experimental and theoretical results (PDF)

\section{AUTHOR INFORMATION}

\section{Corresponding Authors}

Marco Di Giovannantonio - Empa, Swiss Federal Laboratories for Materials Science and Technology, nanotech@ surfaces Laboratory, 8600 Dübendorf, Switzerland; (1) orcid.org/0000-0001-8658-9183; Email: marco.digiovannantonio@empa.ch

Carlo A. Pignedoli - Empa, Swiss Federal Laboratories for Materials Science and Technology, nanotech@surfaces Laboratory, 8600 Dübendorf, Switzerland; 으이.org/00000002-8273-6390; Email: carlo.pignedoli@empa.ch

Klaus Müllen - Max Planck Institute for Polymer Research, 55128 Mainz, Germany; Institute of Physical Chemistry, Johannes Gutenberg University Mainz, 55128 Mainz,
Germany; 이이.org/0000-0001-6630-8786;

Email: muellen@mpip-mainz.mpg.de

Akimitsu Narita - Max Planck Institute for Polymer Research, 55128 Mainz, Germany; Organic and Carbon Nanomaterials Unit, Okinawa Institute of Science and Technology Graduate University, Okinawa 904-0495, Japan; 이이.org/00000002-3625-522X; Email: narita@mpip-mainz.mpg.de

\section{Authors}

Xuelin Yao - Max Planck Institute for Polymer Research, 55128 Mainz, Germany

Kristjan Eimre - Empa, Swiss Federal Laboratories for Materials Science and Technology,nanotech@surfaces Laboratory, 8600 Dübendorf, Switzerland; ㅇo이.org/00000002-3444-3286

José I. Urgel - Empa, Swiss Federal Laboratories for Materials Science and Technology, nanotech@surfaces Laboratory, 8600 Dübendorf, Switzerland; 이이.org/0000-0001-7608-2979

Pascal Ruffieux - Empa, Swiss Federal Laboratories for Materials Science and Technology,nanotech@surfaces Laboratory, 8600 Dübendorf, Switzerland; 이이.org/00000001-5729-5354

Roman Fasel - Empa, Swiss Federal Laboratories for Materials Science and Technology,nanotech@surfaces Laboratory, 8600 Dübendorf, Switzerland; Department of Chemistry and Biochemistry, University of Bern, 3012 Bern, Switzerland; (1) orcid.org/0000-0002-1553-6487

Complete contact information is available at:

https://pubs.acs.org/10.1021/jacs.0c05268

\section{Author Contributions}

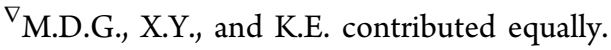

\section{Notes}

The authors declare no competing financial interest.

\section{ACKNOWLEDGMENTS}

This work was supported by the Swiss National Science Foundation under Grant No. 200020_182015, the NCCR MARVEL funded by the Swiss National Science Foundation (51NF40-182892), the Max Planck Society, the European Union's Horizon 2020 research and innovation program under Grant Agreement No. 785219 (Graphene Flagship Core 2), and the Office of Naval Research BRC Program. Computational support from the Swiss Supercomputing Center (CSCS) under Project ID s904 is gratefully acknowledged. We are thankful to E. Paenurk and R. Gershoni-Poranne for fruitful discussion on aromaticity calculations and Lukas Rotach (Empa) for his excellent technical support during the experiments.

\section{REFERENCES}

(1) Beser, U.; Kastler, M.; Maghsoumi, A.; Wagner, M.; Castiglioni, C.; Tommasini, M.; Narita, A.; Feng, X.; Müllen, K. A C216Nanographene Molecule with Defined Cavity as Extended Coronoid. J. Am. Chem. Soc. 2016, 138 (13), 4322-4325.

(2) Cyvin, S. J.; Brunvoll, J.; Cyvin, B. N. Theory of Coronoid Hydrocarbons; Springer Science \& Business Media, 2012.

(3) Miyoshi, H.; Nobusue, S.; Shimizu, A.; Tobe, Y. Non-Alternant Non-Benzenoid Kekulenes: The Birth of a New Kekulene Family. Chem. Soc. Rev. 2015, 44 (18), 6560-6577.

(4) Diederich, F.; Staab, H. A. Benzenoid versus Annulenoid Aromaticity: Synthesis and Properties of Kekulene. Angew. Chem., Int. Ed. Engl. 1978, 17 (5), 372-374. 
(5) Funhoff, D. J. H.; Staab, H. A. Cyclo[d.e.d.e.e.d.e.d.e.e.]Decakisbenzene, a New Cycloarene. Angew. Chem., Int. Ed. Engl. 1986, 25 (8), 742-744.

(6) Hajgató, B.; Deleuze, M. S. Quenching of Magnetism in Hexagonal Graphene Nanoflakes by Non-Local Electron Correlation. Chem. Phys. Lett. 2012, 553, 6-10.

(7) Buttrick, J. C.; King, B. T. Kekulenes, Cycloarenes, and Heterocycloarenes: Addressing Electronic Structure and Aromaticity through Experiments and Calculations. Chem. Soc. Rev. 2017, 46 (1), $7-20$.

(8) Yoneda, K.; Nakano, M.; Inoue, Y.; Inui, T.; Fukuda, K.; Shigeta, Y.; Kubo, T.; Champagne, B. Impact of Antidot Structure on the Multiradical Characters, Aromaticities, and Third-Order Nonlinear Optical Properties of Hexagonal Graphene Nanoflakes. J. Phys. Chem. C 2012, 116 (33), 17787-17795.

(9) Hajgató, B.; Deleuze, M. S.; Ohno, K. Aromaticity of Giant Polycyclic Aromatic Hydrocarbons with Hollow Sites: Super Ring Currents in Super-Rings. Chem. - Eur. J. 2006, 12 (22), 5757-5769.

(10) Kumar, B.; Viboh, R. L.; Bonifacio, M. C.; Thompson, W. B.; Buttrick, J. C.; Westlake, B. C.; Kim, M.-S.; Zoellner, R. W.; Varganov, S. A.; Mörschel, P.; Teteruk, J.; Schmidt, M. U.; King, B. T. Septulene: The Heptagonal Homologue of Kekulene. Angew. Chem., Int. Ed. 2012, 51 (51), 12795-12800.

(11) Majewski, M. A.; Hong, Y.; Lis, T.; Gregoliński, J.; Chmielewski, P. J.; Cybińska, J.; Kim, D.; Stępień, M. Octulene: A Hyperbolic Molecular Belt That Binds Chloride Anions. Angew. Chem., Int. Ed. 2016, 55 (45), 14072-14076.

(12) Zhao, X.-J.; Hou, H.; Ding, P.-P.; Deng, Z.-Y.; Ju, Y.-Y.; Liu, S.H.; Liu, Y.-M.; Tang, C.; Feng, L.-B.; Tan, Y.-Z. Molecular DefectContaining Bilayer Graphene Exhibiting Brightened Luminescence. Sci. Adv. 2020, 6 (9), No. eaay8541.

(13) Palma, C.-A.; Samorì, P. Blueprinting Macromolecular Electronics. Nat. Chem. 2011, 3 (6), 431-436.

(14) Lindner, R.; Kühnle, A. On-Surface Reactions. ChemPhysChem 2015, 16 (8), 1582-1592.

(15) Talirz, L.; Ruffieux, P.; Fasel, R. On-Surface Synthesis of Atomically Precise Graphene Nanoribbons. Adv. Mater. 2016, 28 (29), 6222-6231.

(16) Pozo, I.; Majzik, Z.; Pavliček, N.; Melle-Franco, M.; Guitián, E.; Peña, D.; Gross, L.; Pérez, D. Revisiting Kekulene: Synthesis and Single-Molecule Imaging. J. Am. Chem. Soc. 2019, 141 (39), 1548815493.

(17) Fan, Q.; Martin-Jimenez, D.; Werner, S.; Ebeling, D.; Koehler, T.; Vollgraff, T.; Sundermeyer, J.; Hieringer, W.; Schirmeisen, A.; Gottfried, J. M. On-Surface Synthesis and Characterization of a Cycloarene: C108 Graphene Ring. J. Am. Chem. Soc. 2020, 142 (2), 894-899.

(18) Dias, J. R. Structure and Electronic Characteristics of Coronoid Polycyclic Aromatic Hydrocarbons as Potential Models of Graphite Layers with Hole Defects. J. Phys. Chem. A 2008, 112 (47), 1228112292.

(19) Dias, J. R.; Aihara, J. Antiaromatic Holes in Graphene and Related Graphite Defects. Mol. Phys. 2009, 107 (1), 71-80.

(20) Moreno, C.; Vilas-Varela, M.; Kretz, B.; Garcia-Lekue, A.; Costache, M. V.; Paradinas, M.; Panighel, M.; Ceballos, G.; Valenzuela, S. O.; Peña, D.; Mugarza, A. Bottom-up Synthesis of Multifunctional Nanoporous Graphene. Science 2018, 360 (6385), 199-203.

(21) Yang, H.-X.; Chshiev, M.; Boukhvalov, D. W.; Waintal, X.; Roche, S. Inducing and Optimizing Magnetism in Graphene Nanomeshes. Phys. Rev. B: Condens. Matter Mater. Phys. 2011, 84 (21), 214404.

(22) Mishra, S.; Beyer, D.; Eimre, K.; Kezilebieke, S.; Berger, R.; Gröning, O.; Pignedoli, C. A.; Müllen, K.; Liljeroth, P.; Ruffieux, P.; Feng, X.; Fasel, R. Topological Frustration Induces Unconventional Magnetism in a Nanographene. Nat. Nanotechnol. 2020, 15 (1), 2228.

(23) Ruffieux, P.; Wang, S.; Yang, B.; Sánchez-Sánchez, C.; Liu, J.; Dienel, T.; Talirz, L.; Shinde, P.; Pignedoli, C. A.; Passerone, D.;
Dumslaff, T.; Feng, X.; Müllen, K.; Fasel, R. On-Surface Synthesis of Graphene Nanoribbons with Zigzag Edge Topology. Nature 2016, 531 (7595), 489-492.

(24) Di Giovannantonio, M.; Deniz, O.; Urgel, J. I.; Widmer, R.; Dienel, T.; Stolz, S.; Sánchez-Sánchez, C.; Muntwiler, M.; Dumslaff, T.; Berger, R.; Narita, A.; Feng, X.; Müllen, K.; Ruffieux, P.; Fasel, R. On-Surface Growth Dynamics of Graphene Nanoribbons: The Role of Halogen Functionalization. ACS Nano 2018, 12 (1), 74-81.

(25) Clair, S.; de Oteyza, D. G. Controlling a Chemical Coupling Reaction on a Surface: Tools and Strategies for On-Surface Synthesis. Chem. Rev. 2019, 119 (7), 4717-4776.

(26) Cai, J.; Ruffieux, P.; Jaafar, R.; Bieri, M.; Braun, T.; Blankenburg, S.; Muoth, M.; Seitsonen, A. P.; Saleh, M.; Feng, X.; Müllen, K.; Fasel, R. Atomically Precise Bottom-up Fabrication of Graphene Nanoribbons. Nature 2010, 466 (7305), 470-473.

(27) Di Giovannantonio, M.; Contini, G. Reversibility and Intermediate Steps as Key Tools for the Growth of Extended Ordered Polymers via On-Surface Synthesis. J. Phys.: Condens. Matter 2018, 30, 093001.

(28) Fan, Q.; Wang, T.; Dai, J.; Kuttner, J.; Hilt, G.; Gottfried, J. M.; Zhu, J. On-Surface Pseudo-High-Dilution Synthesis of Macrocycles: Principle and Mechanism. ACS Nano 2017, 11 (5), 5070-5079.

(29) Gross, L.; Mohn, F.; Moll, N.; Liljeroth, P.; Meyer, G. The Chemical Structure of a Molecule Resolved by Atomic Force Microscopy. Science 2009, 325 (5944), 1110-1114.

(30) Bieri, M.; Treier, M.; Cai, J.; Aït-Mansour, K.; Ruffieux, P.; Gröning, O.; Gröning, P.; Kastler, M.; Rieger, R.; Feng, X.; Müllen, K.; Fasel, R. Porous Graphenes: Two-Dimensional Polymer Synthesis with Atomic Precision. Chem. Commun. 2009, No. 45, 6919.

(31) Jiang, D.; Cooper, V. R.; Dai, S. Porous Graphene as the Ultimate Membrane for Gas Separation. Nano Lett. 2009, 9 (12), 4019-4024.

(32) Du, A.; Zhu, Z.; Smith, S. C. Multifunctional Porous Graphene for Nanoelectronics and Hydrogen Storage: New Properties Revealed by First Principle Calculations. J. Am. Chem. Soc. 2010, 132 (9), 2876-2877.

(33) Pedersen, T. G.; Flindt, C.; Pedersen, J.; Mortensen, N. A.; Jauho, A.-P.; Pedersen, K. Graphene Antidot Lattices: Designed Defects and Spin Qubits. Phys. Rev. Lett. 2008, 100 (13), 136804.

(34) Liu, C.; Sandoval-Salinas, M. E.; Hong, Y.; Gopalakrishna, T. Y.; Phan, H.; Aratani, N.; Herng, T. S.; Ding, J.; Yamada, H.; Kim, D.; Casanova, D.; Wu, J. Macrocyclic Polyradicaloids with Unusual Super-Ring Structure and Global Aromaticity. Chem. 2018, 4 (7), $1586-1595$

(35) Fias, S.; Fowler, P. W.; Delgado, J. L.; Hahn, U.; Bultinck, P. Correlation of Delocalization Indices and Current-Density Maps in Polycyclic Aromatic Hydrocarbons. Chem. - Eur. J. 2008, 14 (10), 3093-3099. 